\title{
Grid insensitive modelling of convective heat transfer fluxes in CFD simulations of medium-scale pool fires
}

Georgios Maragkos ${ }^{a^{*}}$, Bart Merci ${ }^{a}$

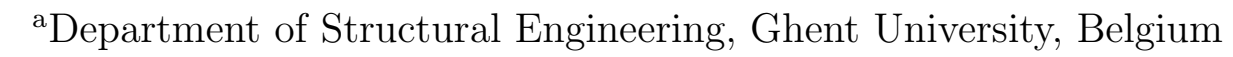

Georgios.Maragkos@UGent.be

${ }^{*}$ Corresponding author

7 Highlights:

- Different approaches for calculating the convective heat fluxes at the pool surface of alcohol fuels are investigated.

- The predicted convective heat fluxes are highly grid dependent when calculated based on the first grid cell temperatures.

- Calculation of the convective heat fluxes based on the stagnant film theory, using an average flame temperature, is the best approach and only slightly grid sensitive.

\begin{abstract}
:
Focusing on convective heat transfer modelling of pool fires, different approaches for calculating the convective heat fluxes at the pool surface have been employed, namely to resolve them; model them based on Nusselt correlations for forced or natural convection; model them based on the stagnant film theory. Large eddy simulations of medium-scale, 30 $\mathrm{cm}$ in diameter, alcohol pool fires (methanol, ethanol and acetone) are conducted and compared against experimental convective and radiative heat flux measurements. Overall, the best approach is the one based on the stagnant film theory which is only slightly grid sensitive, and provides satisfactory predictions for all the different fuels tested. The predictions using the other approaches are highly grid dependent, due to a fuel rich region formed just above the pool surface when the grid size is refined, which greatly influence the calculation of the convective heat fluxes using the first grid cell values.
\end{abstract}

Keywords: modelling; heat transfer; CFD; convection; pool fire

\section{Introduction}

Numerical modelling of boundary layer flows [1] within the context of fire applications remains very challenging and accurate prediction of heat transfer is often essential in fire scenarios. The intricate coupling of the different modelling aspects (i.e., turbulence, combustion, radiation, soot) involved in the gas phase cannot be easily decoupled and strongly affects heat transfer modelling. Even though some challenges still remain (e.g., related to soot modelling), gas phase fire modelling has nowadays reached a certain level of maturity which enables fire research to focus on more complex fire modelling aspects such 
as boundary layer flows involving combustion. Within this context, accurate modelling of convective and radiative heat transfer of boundary layer flows is an essential aspect in fire modelling which provides a solid foundation for further modelling of more complex fire scenarios (e.g., involving pyrolysis and flame spread, liquid fuel evaporation, etc.). The fuel gasification rate (i.e., either pyrolysis or liquid evaporation) is determined by a heat feedback mechanism which depends on convective and radiative heat transfer [2]. The feedback due to convection is through the convection boundary layer above the fuel surface generated by buoyancy while the radiative feedback is due to radiation from the flames, accurate prediction of which is challenging. Firstly, high grid resolutions are typically required in order to resolve the small-scale flow features occurring in the boundary layers which make numerical simulations computationally expensive. Secondly, fires typically involve low-to-moderate Reynolds number flows for which the classical theory of turbulent boundary layers is not strictly applicable [3]. Finally, multi-physics phenomena are involved, e.g., mass transpiration effects, turbulence, combustion and radiation, that require accurate near-surface modelling.

From employing a boundary layer analysis, for either momentum-driven or natural convection flows, it is well established that grid resolutions in the order of mm are needed in order to have accurate predictions of convective heat transfer $[4,5]$. Such grid resolutions, even with the advances of today's computer software and technology, are often prohibitive for typical fire scenarios involving wide range of time and length scales. Most of the Computational Fluid Dynamics (CFD) codes, often employed to model fire scenarios, have been relying on modelling convective heat transfer using either wall functions $[6,7,8]$ or experimentally derived correlations [6] for specific types of flows. More specifically, convective heat transfer is often modelled by employing Nusselt correlations either for natural or forced convection since the alternative approach (i.e., use of wall functions) typically requires too fine grid resolutions in the near-wall region. The limitations of such approaches can be significant since they are not easily applicable to fire scenarios and often they ignore much of the different physics involved. The main disadvantages from the use of such correlations are that they have been developed for non-reacting flows, considering either iso-thermal surfaces or constant surface fluxes, and that they ignore mass transpiration effects. The limitations related to the application of such approaches to CFD codes are in fact even greater since there is no well-defined free stream flow to be used as input for these correlations. Rather, the first grid-value is used, and the resulting heat transfer rates have been shown to be highly grid dependent (i.e., on the choice of the first grid size). It is worth noting that uncertainties up to $25 \%$ have been reported in the heat transfer coefficients based on these experimental correlations depending on the free-stream properties and the surface roughness alone [9]. It is obvious that there are significant uncertainties from the application of the aforementioned correlations in their current form to fire scenarios involving liquid pool fires and/or flammable solid walls. Some of the limitations mentioned are difficult to be circumvented. Nevertheless, significant improvements are needed in order to have accurate convective heat transfer predictions over a wide range of grid sizes. 
Convective heat transfer has been reported to be important for small to medium-scale pool fires with diameters of up to $D<0.3 \mathrm{~m}[10,11]$ with its impact often comparable to that of radiative heat transfer. The influence of convective heat transfer diminishes with increasing pool diameters for $0.3<D<1.0 \mathrm{~m}$ until radiative heat transfer becomes the dominant heat feedback mechanism for pool fires with diameters $D>1.0 \mathrm{~m}$ [12] due to the 'blowing' effect caused by vapor mass flux leaving the fuel surface [13]. Therefore, the influence of convective heat transfer modelling cannot be easily disregarded in scenarios involving medium-scale pool fires and it can have a direct impact in the resulting mass burning rates.

Fire safety engineers often employ fire modelling, through the use of CFD, in order to simulate scenarios involving liquid fuel evaporation. Therefore, accurate modelling of convective heat transfer in pool fires over a wide range of grid sizes is essential. The present work focuses on convective heat transfer modelling and aims to investigate the accuracy of some of the typically used approaches in fire modelling. For this reason, Large Eddy Simulations (LES) of various medium-scale pool fires $(D=0.3 \mathrm{~m})$ involving alcohol fuels are conducted. The focus is on alcohol fuels in order to minimize uncertainties related to modelling of highly sooty fuels. The numerical predictions are compared against well characterized experiments. Focus is mainly on comparing the predicted heat fluxes at the pool surface since accurate prediction of these is a pre-requisite for accurate modelling of liquid fuel evaporation as well (i.e., through the heat feedback mechanism).

\section{Modelling}

The CFD code FireFOAM 2.2.x [14], originally developed by FM Global, is employed here. The code uses a fully compressible flow formulation and solves the Navier-Stokes equations, along with transport equations for species mass fractions and sensible enthalpy, using Favre-filtered quantities, and employing a unity Lewis number assumption. A detailed presentation of the code and the sub-models has been previously reported by the authors (e.g., [15]) so only a general overview is outlined here. Turbulence is modelled through the dynamic Smagorinsky model with a variable Prandtl number formulation calculated based on a dynamic procedure. Chemistry is considered to be infinitely fast and turbulence-chemistry interactions are modelled with the Eddy Dissipation Model (EDM) considering a turbulent mixing time scale and a model constant of $C_{E D M}=2$. Radiation is modelled through the finite volume discrete ordinates model (fvDOM) with the absorption/emission modelled through the weighted sum of gray gases model (WSGGM). The only participating gases considered are $\mathrm{CO}_{2}$ and $\mathrm{H}_{2} \mathrm{O}$. The path length is calculated dynamically during the simulation as $L=3.6 \mathrm{~V} / \mathrm{A}$. The volume, $\mathrm{V}$, is calculated by summing all the cell volumes where reaction takes place and by assuming a conical flame shape, the corresponding surface area, $A$, is calculated from simple geometric formulas.

Focusing on modelling convective heat transfer, different methods for calculating the convective heat fluxes at the pool surface are employed, an outline of which is summarized below. 
- Method 1:

$$
\dot{q}_{c o n v}^{\prime \prime}=\rho \alpha_{e f f} c_{p} \frac{d T}{d n}
$$

where $\alpha_{e f f}=\alpha+\alpha_{s g s}, n$ denotes the direction normal to the pool surface and $d T / d n$ is the temperature gradient based on the first grid cell temperature and the pool surface temperature (i.e., set to the boiling temperature of the fuel). The molecular thermal diffusivity, $\alpha$, is temperature dependent while the sub-grid scale thermal diffusivity is calculated as $\alpha_{s g s}=\mu_{s g s} / P r_{t}$. This method [6] essentially does not involve any modelling and is suitable for well resolved simulations where the grid sizes are small enough to accurately resolve the boundary layer flow. In this case, a Neumann boundary condition is applied for $\alpha_{s g s}$ at the pool surface (i.e., zero gradient).

- Method 2:

$$
\dot{q}_{\text {conv }}^{\prime \prime}=h\left(T-T_{\text {surf }}\right)
$$

where the convection coefficient, $h$, is calculated from correlations based on the Nusselt number for forced convection over horizontal plates [16]:

$$
N u=\frac{h L}{k}=0.664 \operatorname{Re}^{1 / 2} \operatorname{Pr}^{1 / 3}, \quad R e<R e_{\text {crit }}
$$

$$
N u=\frac{h L}{k}=0.037 \operatorname{Re}^{4 / 5} \operatorname{Pr}^{1 / 3}, \quad R e \geq R e_{\text {crit }}
$$

accounting for both laminar and turbulent flow conditions, respectively. In this case, $k$ is the conductivity of the gas phase, $L$ is the characteristic length of the problem (i.e., $L=D$ where $D$ is the pool diameter), $R e=|U| L / \nu$ is the Reynolds number, $\operatorname{Pr}$ is the Prandtl number and the critical Reynolds number for flow over plates is taken to be $R e_{\text {crit }}=5 \times 10^{5}$. The temperature, $T$, is based on the first grid cell value while the temperature at the pool surface, $T_{\text {surf }}$, is set to the boiling temperature of the fuel (i.e., $T_{\text {surf }}=T_{\text {boil }}$ ). The calculation of the Reynolds number considers the magnitude of the local grid cell velocity vector while the kinematic viscosity, $\nu$, and thermal conductivity, $k$, (used in both Methods 2 and 3) are temperature dependent and are based on the first grid cell values.

- Method 3:

$$
\dot{q}_{\text {conv }}^{\prime \prime}=h\left(T-T_{\text {surf }}\right)
$$

where the convection coefficient, $h$, is calculated from correlations based on the Nusselt number for natural convection over horizontal plates [17]:

$$
N u=\frac{h L}{k}=0.54 R a^{1 / 4}, \quad 10^{4} \leq R a<10^{7}
$$

$$
N u=\frac{h L}{k}=0.15 R a^{1 / 3}, \quad 10^{7} \leq R a \leq 10^{11}
$$


accounting for both laminar and turbulent flow conditions, respectively. The Rayleigh number is defined as $R a=G r P r$ with the Grashof number calculated as:

$$
G r=\frac{g \beta\left(T-T_{\text {surf }}\right) L^{3}}{\nu^{2}}
$$

where $g$ is the gravitational acceleration, $\beta=1 / T$ is the volumetric expansion coefficient while the characteristic length for natural convection is $L=A_{s} / P$ where $A_{s}$ and $P$ are the surface area and perimeter of the pool surface, respectively. The temperature, $T$, is based on the first grid cell value while the temperature at the pool surface, $T_{\text {surf }}$, is set to the boiling temperature of the fuel (i.e., $T_{\text {surf }}=T_{\text {boil }}$ ).

- Method 4:

$$
\dot{q}_{\text {conv }}^{\prime \prime}=\frac{h}{c_{p}}\left[\left(\frac{\chi_{a}-\chi_{r}}{\chi_{a}}\right) Y_{O_{2}, \infty} \Delta H_{O_{2}}-c_{p}\left(T_{\text {surf }}-T_{\infty}\right)\right] \frac{\dot{m}^{\prime \prime} c_{p} / h}{\exp \left(\dot{m}^{\prime \prime} c_{p} / h\right)-1} R
$$

where $\chi_{a}$ and $\chi_{r}$ are the combustion efficiency and (global) radiative fraction of the fuel, respectively, $Y_{O_{2}, \infty}$ is the ambient oxygen mass fraction, $\Delta H_{O_{2}}$ is the heat of combustion per gr of $\mathrm{O}_{2}$ consumed, $c_{p}$ is the heat capacity, the pool surface temperature is set to the boiling tempertaure of the fuel (i.e., $T_{\text {surf }}=T_{\text {boil }}$ ), $T_{\infty}=293 \mathrm{~K}$ is the ambient temperature and $\dot{m}^{\prime \prime}$ is the fuel mass evaporation rate per unit area. This expression essentially stems from the stagnant film theory $[12,19]$ which describes liquid fuel evaporation due to convective heat transfer from nearby flames which accounts for the 'blowing' effect (i.e., reduction of the convective heat fluxes due to mass transpiration). The convective heat transfer coefficient, $h$, is calculated from correlations based on the Nusselt number for natural convection over a flat plate (i.e., Eqs (6) and (7)) while the Grashof number from Eq. (8) considering not the first grid cell temperatures (like in methods 1-3) but an average flame temperature based on the values reported by Hamins et al. [20] (i.e., $T=1114 \mathrm{~K}$ for methanol, $T=1034 \mathrm{~K}$ for ethanol and $T=1042 \mathrm{~K}$ for acetone). This approach essentially tries to minimize any grid dependency in the calculation of the convective heat fluxes that could occur unless fine grid sizes are used close to the pool surface to resolve the steep temperature gradients (i.e., need for accurately resolving the boundary layer). These errors in the resulting convective heat fluxes would essentially be stemming from not accurately predicting the temperature difference either directly, (i.e., $\Delta T$ in Eqs (1), (2) and (5)) or indirectly (i.e., $\Delta T$ in Eq (8)). The parameter $R$ is used here as a correction in the convective heat flux profile since Eq. (9) will give a relatively constant profile over the pool surface. Since method 4 does not depend on the local temperature directly (i.e., an average flame temperature is used in the calculation of the Grashof number), any spatial variation will only be due to the calculation of $c_{p}$ and $h$ (through the kinematic viscosity $\nu$ and thermal conductivity $k$ ) which are based on the first grid cell values. A simple profile in the form of $R=1-(2 r / D)^{5}$ is employed, where $r$ is the radial distance and $D$ the pool diameter. Given the overall trends of the experimental heat flux profiles [21] (i.e., decreasing fluxes towards the pan rim for methanol and ethanol and relatively constant 
profile over the pan diameter for acetone), this correction has been only applied for the methanol and ethanol pool fires. It is worth noting an average flame temperature could have been calculated during the simulations followed by a direct coupling of the predicted radiative fractions instead of prescribing constant values a-priori. Even though this way the simulations with method 4 would be more 'predictive' they would also be more grid dependent.

Modelling of convective heat transfer based on the stagnant film theory (i.e., equation (9)) has been performed in the past e.g., through global modelling focusing on predicting the mass loss rates of pool fires (e.g. [19, 20]), for determining the convective heat fluxes from pool fire experiments (e.g., [22]) and to some extent in numerical simulations for determining the fuel mass loss rates in mechanically ventilated compartments (e.g., $[23,24])$. Nevertheless, this approach has not been extensively used in CFD simulation of pool fires nor has been extensively compared to other methods.

\section{Test cases}

Focus is mainly given to modelling cases involving alcohol fuels in order to minimize any additional uncertainties related to soot modelling. For this reason, the experimental tests cases that will be used for comparative purposes are the medium-scale pool fire experiments reported in [21], a brief overview of which is given in Table 1. The experiments involved a circular stainless-steel pan with an inner diameter of approximately $0.3 \mathrm{~m}$ and three different alcohol fuels (i.e., methanol, ethanol and acetone). The bottom of the pan was water-cooled at an almost constant temperature of about $20^{\circ} \mathrm{C}$ while the fuel level was set $1 \mathrm{~cm}$ below the top of the burner. The experimentally measured total heat fluxes for the three fuels will be used here for comparative purposes. The experimental uncertainties in the measurements of the total heat fluxes were reported to be in the order of $20 \%$. Additional experimental data of radiative heat fluxes for methanol pool fires of the same diameter (but with a $5 \mathrm{~mm}$ rim height) from [10] have also been considered since the combined set of data from $[10,21]$ can give an estimate of the convective heat fluxes for the methanol pool fire case.

\section{Numerical setup}

A cylindrical computational domain of $1.5 \mathrm{~m} \mathrm{x} 1.8 \mathrm{~m}$ is used in the simulations. The base mesh consists of approximately $2 \mathrm{~cm}$ cells on the centerline stretched towards the side and top boundaries (ratio of initial to final grid size is 1.5). A refinement strategy is then employed in order to have a good grid resolution in the near-field region of the pool fires. A cylindrical box having dimension of $0.9 \mathrm{~m} \times 0.9 \mathrm{~m}$ refines the mesh to $1 \mathrm{~cm}$ while a second cylindrical box of dimensions $0.6 \mathrm{~m} \times 0.6 \mathrm{~m}$ refines the mesh to $0.5 \mathrm{~cm}$. The total number of cells is then approximately 650000. A sensitivity study with all the three different grid sizes is included in the study. The experimentally reported pan rim height of $1 \mathrm{~cm}$ was considered in the simulations apart from the $2 \mathrm{~cm}$ case where the height of the rim was set 
Table 1: Overview of the different experimental test cases [21] considered.

\begin{tabular}{lccc}
\hline Fuel & Methanol & Ethanol & Acetone \\
\hline Chemical formula & $\mathrm{CH}_{3} \mathrm{OH}$ & $\mathrm{C}_{2} \mathrm{H}_{5} \mathrm{OH}$ & $\left(\mathrm{CH}_{3}\right)_{2} \mathrm{CO}$ \\
\hline Pan diameter $(\mathrm{m})$ & 0.3 & 0.3 & 0.3 \\
\hline $\mathrm{T}_{\text {boil }}\left({ }^{\circ} \mathrm{C}\right)$ & 64.7 & 78.3 & 56.3 \\
\hline $\mathrm{HRR}(\mathrm{kW})$ & 19.2 & 31.0 & 38.1 \\
\hline$\chi_{r}(-)$ & 0.19 & 0.21 & 0.27 \\
\hline$\chi_{a}(-)$ & $\approx 1.0$ & $\approx 1.0$ & $\approx 1.0$ \\
\hline$\Delta H_{\mathrm{O}_{2}}(\mathrm{~kJ} / \mathrm{g})[18]$ & 13.22 & 12.88 & 14.0 \\
\hline
\end{tabular}

to $2 \mathrm{~cm}$. The influence of the pan rim has been shown to be an important aspect when modelling pool fire cases in the past since the flames remain attach to it. Given this aspect and the fact that the rim in the experiments is $1 \mathrm{~cm}$, prohibits the use of grid sizes much coarser than $1 \mathrm{~cm}$.

In order to avoid any uncertainty related to liquid evaporation modelling, the fuels are considered to have fully evaporated, hence, the fuel mass flow rate is assigned at the pool surface, accounting for both convective and diffusive mass fluxes. The temperature at the pool surface is set to the equivalent boiling temperature of the different fuels considered (see Table 1) while the pan rim is modelled as isothermal at $20^{\circ} \mathrm{C}$ since the pan was water-cooled during the experiments. The ambient temperature and pressure are $293 \mathrm{~K}$ and $101325 \mathrm{~Pa}$, respectively. All numerical simulations are set to run for $35 \mathrm{sec}$ with a varying time step, limited by a maximum Courant number of 0.9 , with averaging occurring over the last $30 \mathrm{sec}$. The equations are advanced in time using a second order backward scheme. A second order filtered linear scheme is employed for the convective terms in the momentum equations while a TVD scheme (i.e., limitedLinear) for scalar transport.

\section{Results}

In this section, the predicted heat fluxes from the numerical simulations are compared against experimental data for different alcohol fuels [10, 21]. It is worth noting that the experimental values presented below are expected to be slightly lower because the measurements have been performed $3 \mathrm{~mm}$ above the pan rim $(13 \mathrm{~mm}$ above the fuel surface) and not directly at the pool surface due to experimental limitations. These lower heat flux values are essentially due to fuel vapor absorption and differences in the velocity field which can affect convective heat transfer [21]. Experimental uncertainties (where available) have been included in the figures as error bars. 


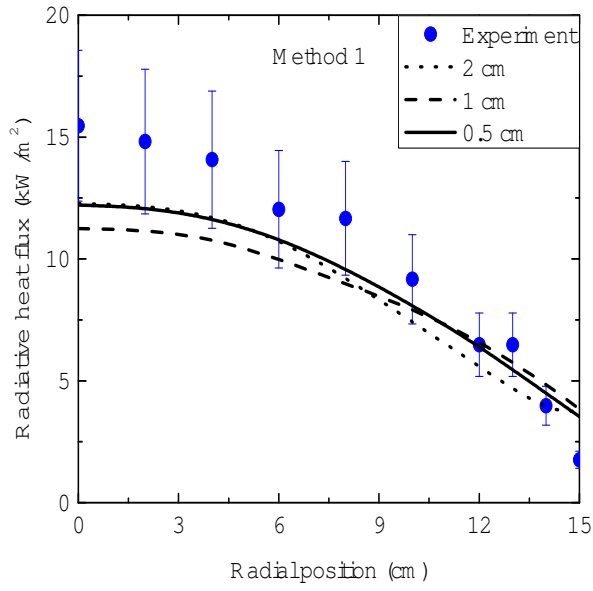

(a)

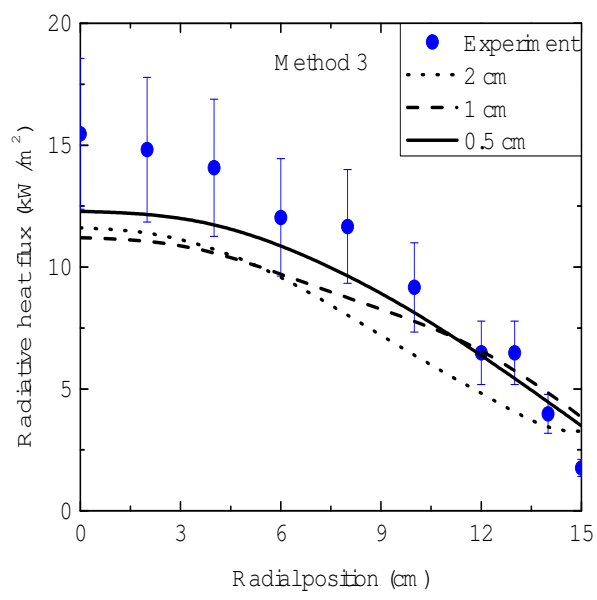

(c)

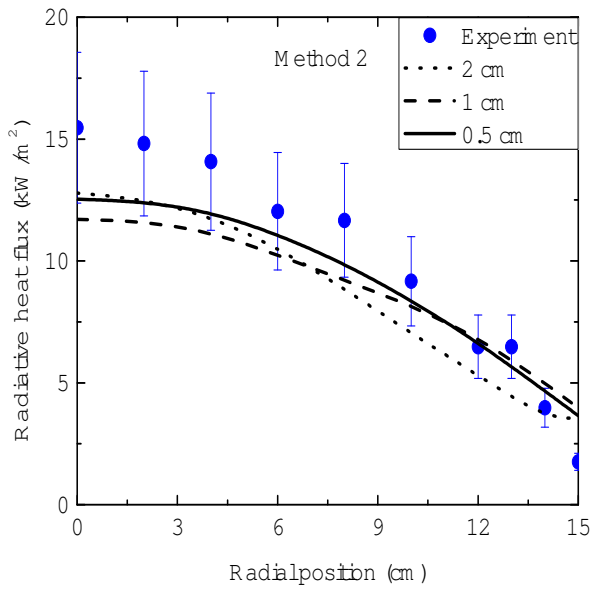

(b)

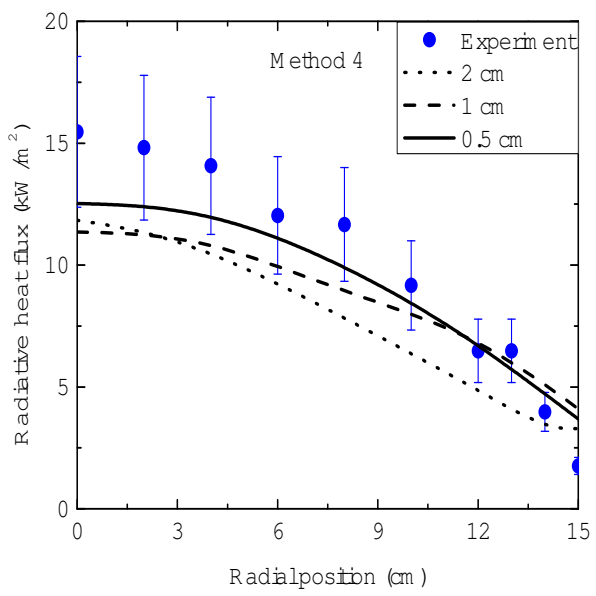

Fig. 1. Average radiative heat fluxes at the methanol $\left(\mathrm{CH}_{3} \mathrm{OH}\right)$ pool surface as a function of grid size with (1) method 1, (b) method 2, (c) method 3 and (d) method 4.

\subsection{Methanol}

The predicted radiative heat fluxes at the methanol $\left(\mathrm{CH}_{3} \mathrm{OH}\right)$ pool surface, shown in Figure 1, match the experimental values relatively well and remain close to the experimental uncertainty with, overall, no significant grid dependency observed. As expected, the different methods employed for calculating the convective heat fluxes at the pool surface, do not have any significant influence on the predicted radiative heat fluxes. Any discrepancies in the predicted radiative heat fluxes, particularly on the coarsest grid size (i.e., $2 \mathrm{~cm}$ ), are mainly attributed to the under-prediction of the radiative fractions in the simulations. Some differences due to the influence of a higher pan rim in the case of the $2 \mathrm{~cm}$ grid size are also to be expected which could affect heat transfer at the pool surface for all the cases examined. 


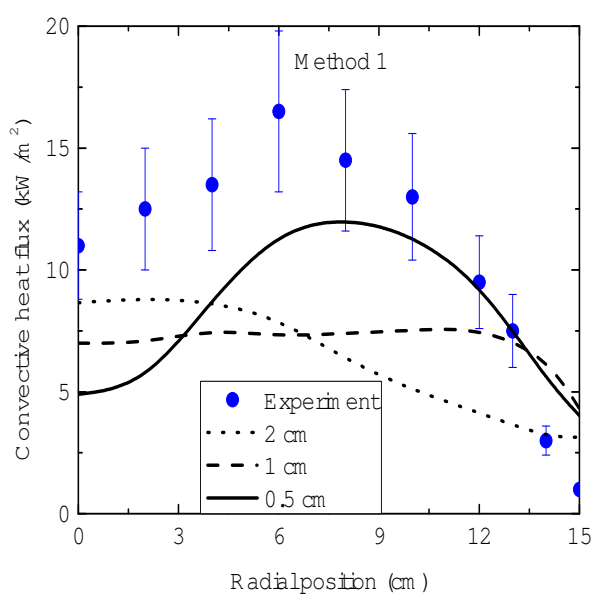

(a)

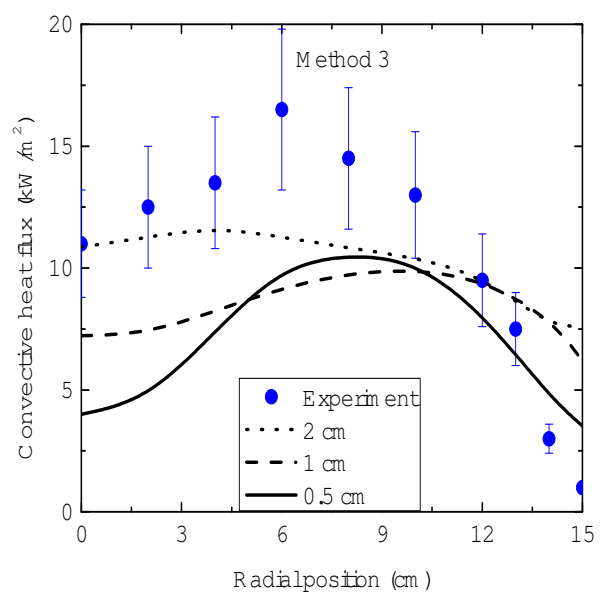

(c)

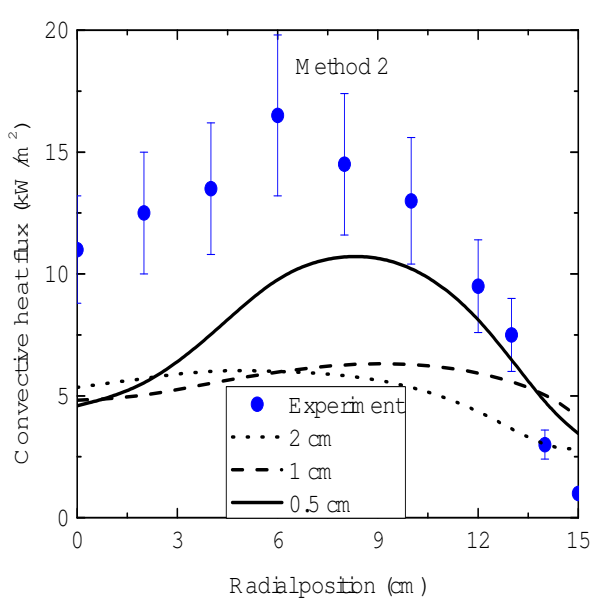

(b)

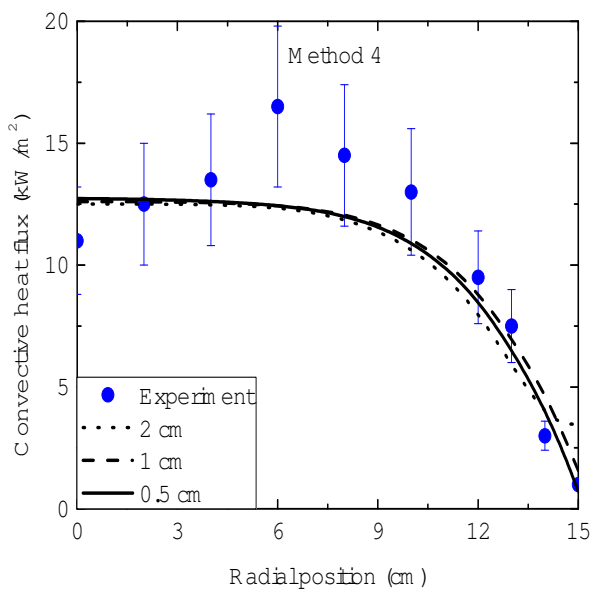

Fig. 2. Average convective heat fluxes at the methanol $\left(\mathrm{CH}_{3} \mathrm{OH}\right)$ pool surface as a function of grid size with (1) method 1, (b) method 2, (c) method 3 and (d) method 4.

The predicted radiative fraction (calculating the convective heat fluxes with method 4) for the $2 \mathrm{~cm}, 1 \mathrm{~cm}$ and $0.5 \mathrm{~cm}$ test cases were $0.133,0.167$ and 0.170 , respectively, while the experimental was $0.19 \pm 26 \%$ [21]. Overall, the radiative fractions, are fairly well predicted $( \pm 10 \%)$ when compared to the experimentally reported value, mainly for the $1 \mathrm{~cm}$ and 0.5 $\mathrm{cm}$ grid size cases. However, the predictions for the $2 \mathrm{~cm}$ grid size case are under-predicted by approximately $30 \%$. Given the fact that the predicted radiative heat fluxes (and radiative fractions), greatly depend on the predicted flame temperatures in the simulations it is rather difficult to avoid such grid dependencies when coarser grids are employed. Nevertheless, the predictions related to radiative heat transfer with the usage of the WSGGM model are satisfactory in this case.

The predicted convective heat fluxes at the methanol pool surface, with the four different 
methods employed in the simulations, are presented in Figure 2. Overall, a strong grid dependency is observed in the predicted fluxes with methods 1-3, and in most cases a decrease of the convective heat fluxes on the centerline and close to the pool edges is observed. This result is in fact not very surprising. When relatively coarse grids are employed (i.e., few cells across the pool surface), fuel and oxidizer mix rapidly and result in high fuel reaction rates in the first grid cells just above the pool surface. As the grid size is refined (i.e., more cells across the pool surface), a fuel rich region starts to develop just above the pool surface (i.e., more profound on the centerline and at the edges of the pool) resulting in low fuel reaction rates just above the pool surface (see Figure 3). This aspect results in higher temperature differences (i.e., based on the first grid cell and the surface temperature) in the case of coarser cells as opposed to when finer grid sizes are employed. This decrease of $\Delta T$ as the grid size is refined does not only affect the calculation of the convective heat flux directly but also indirectly through the calculation of the Grashof number in the case of method 3. This aspect is not encountered, to a big extent, with method 2 since the calculation of the convective heat transfer coefficient does not explicitly depend on temperature, rather on the (local) first grid cell velocity which remains relatively constant around the centerline. Yet, an obvious grid dependency in the predictions is observed at the other radial locations. On the other hand, this grid dependency is not seen with method 4 in which an, overall, average flame temperature is used in the simulations. It is worth noting that the grid size dependency in $\Delta T$, due to the development of fuel rich regions just above the pool surface (previously also reported by Sikanen et al. [3]), seen with methods 1-3 will not be present in other scenarios, e.g., involving boundary layer flows over inert surfaces or flame spread over flammable walls. In such scenarios, the grid refinement will result in better resolution of the boundary layer flow and will more accurately capture the temperature gradient near the wall, hence, an increase of the convective heat fluxes with decreasing grid size is expected.

Strongly reducing the temperature dependency of the calculation of the convective heat fluxes is the main reason as to why relatively accurate heat flux predictions are obtained with method 4, even for coarser grids (i.e., $2 \mathrm{~cm}$ ). More specifically, the expression used for calculating the convective heat fluxes (based on the stagnant film theory) does not explicitly involve a temperature difference. Additionally, any temperature dependency that would arise from using the first grid cell temperature values in the calculation of the Grashof number is avoided by considering an overall flame temperature. Any possible grid dependency then only enters in the evaluation of other variables such as e.g., the heat capacity, $c_{p}$, and the kinematic viscosity, $\nu$, which appears to be less crucial compared to the temperature calculation. A similar approach could potentially also be applied to model convective heat transfer in flame spread scenarios which could be interesting to investigate in the future.

Both methods 2 and 3, namely the usage of either forced or natural convection approach, used for modelling convective heat transfer at the pool surface did not perform well. Considering that the flow velocities at the base of the pool fires are significantly low (i.e., 


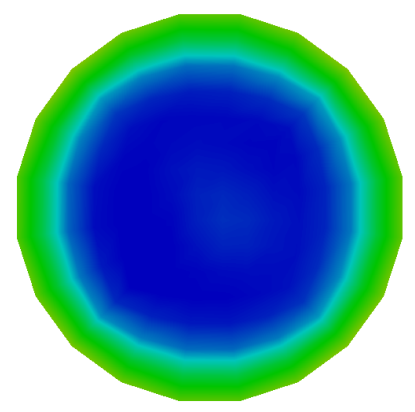

(a)

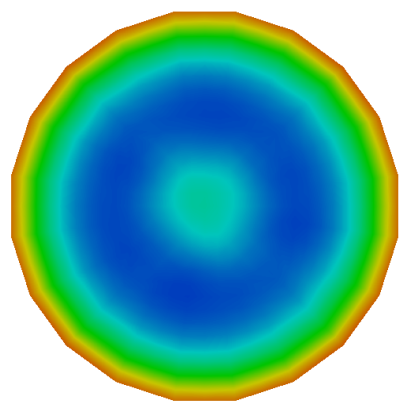

(b)

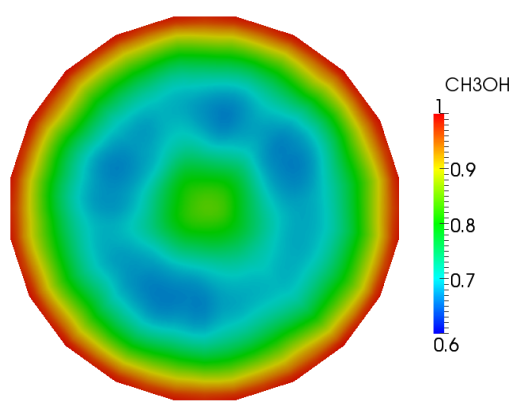

(c)

Fig. 3. Average methanol $\left(\mathrm{CH}_{3} \mathrm{OH}\right)$ mass fraction at the pool surface with grid sizes of (1) $2 \mathrm{~cm}$, (b) $1 \mathrm{~cm}$ and (c) $0.5 \mathrm{~cm}$, calculating the convecting heat fluxes with method 4 .

in the order of a few $\mathrm{cm} / \mathrm{s}$ ) perhaps the consideration of a forced convection problem is not totally justified and suitable as opposed to employing the natural convection approach. Nevertheless, the results from both methods 2 and 3 were in fact very comparable to the predictions of method 1 , where the convective heat fluxes were not modelled rather an attempt was made to resolve them. These results raise some questions regarding the validity of these approaches, namely whether the boundary layer analysis, in the case where very low velocities are present for forced convection problems, is still valid and when the first grid size values are used as free stream values in the correlations. Based on the present study, no significant advantage was observed from the usage of methods 2 and 3 as opposed to method 1 when modelling convective heat transfer at the pool fire surface. It is interesting to note that relatively accurate predictions for the convective heat fluxes were obtained with method 3 coincidentally for the $2 \mathrm{~cm}$ grid size case but were severely under-predicted when the grid sizes were refined for the reasons explained above. Therefore, care must be taken because the convective heat flux predictions in the case of pool fires, based on the first grid cell values, can be highly grid dependent.

Method 4 performed, overall, better when compared to any of the other approaches employed both in terms of accuracy and performance over different grid sizes. Both the maximum values and the profiles (through the usage of the profile correction) of the convective heat fluxes were well predicted. The fact that method 4 does not explicitly involve a temperature difference in the calculation of the convective heat fluxes results in predictions which are less sensitive to the grid size. The usage of a single average flame temperature, and not the first grid cell temperature, in the calculation of the Grashof number also contributes to this aspect. Even though not used in the present study, it is worth noting that method 4 does not necessarily require an a priori prescription of the radiative fraction but could be coupled to the predictions of $\chi_{r}$ from the WSGGM model. This coupling would make the approach more predictive but would also increase the grid dependency of the method particularly when coarse grid sizes are used and the radiative fractions are under-predicted. The deficiencies of methods 1-3 are in fact not only restricted to the methanol pool fire examined here but are applicable to all pool fire 


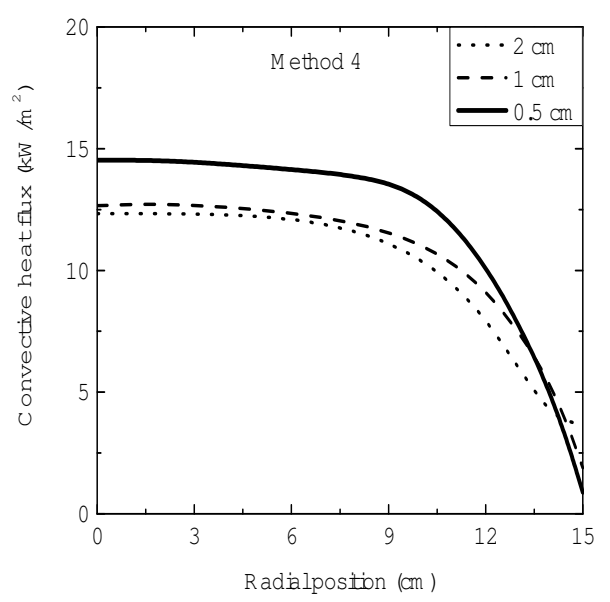

(a)

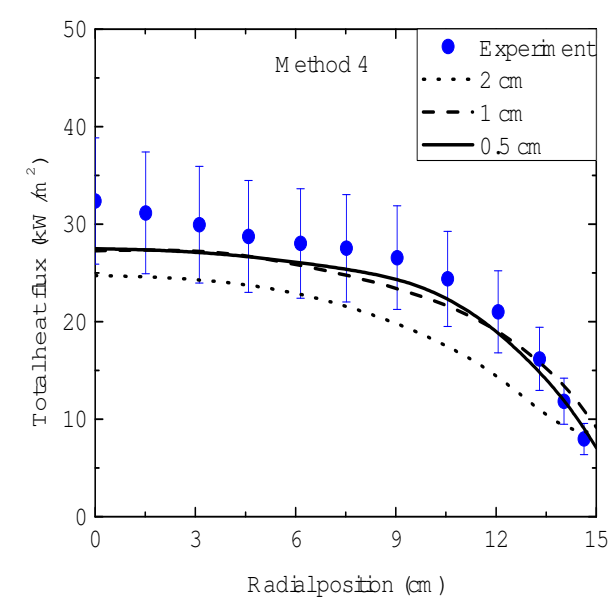

(b)

Fig. 4. Average (a) convective and (b) total heat fluxes at the ethanol $\left(\mathrm{C}_{2} \mathrm{H}_{5} \mathrm{OH}\right)$ pool surface with method 4.

scenarios, irrespective of the fuel type. A significant advantage of method 4 is that it will be able to account for any changes in the convective heat fluxes over a wide range of scenarios, e.g., when different pan diameters (i.e., through changes in $\dot{m}^{\prime \prime}$ ) and different fuels (i.e., through changes in $\chi_{r}$ ) with relatively small grid dependency in the predictions. For the remaining of the paper reporting on the numerical predictions for ethanol and acetone, results with method 4 are presented, since it performed superior compared to the other approaches, along with results with method 1 for comparison purposes. Overall, the predictions with methods 2 and 3 followed the same trends and exhibited the same deficiencies (e.g., strong grid dependency) as the ones reported for the methanol test case.

\subsection{Ethanol}

The predicted convective and total heat fluxes, as a function of grid size, in the case of the ethanol pool fire $\left(\mathrm{C}_{2} \mathrm{H}_{5} \mathrm{OH}\right)$ are presented in Figures 4-5. The relatively small grid dependency, previously seen in the predicted convective heat fluxes for the methanol pool fire with method 4, is observed here for ethanol as well. The numerical predictions are close to the experimental profiles of the total heat fluxes and in most cases remain within the experimental uncertainty. The small discrepancies for the $2 \mathrm{~cm}$ grid size case can again be partially attributed to the under-prediction of the radiative fractions in the simulations. The predicted radiative fraction (calculating the convective heat fluxes with method 4) for the $2 \mathrm{~cm}, 1 \mathrm{~cm}$ and $0.5 \mathrm{~cm}$ test cases were $0.151,0.212$ and 0.230 , respectively, while the experimental was $0.21 \pm 26 \%$ [21]. In this case, an under-prediction of approximately $30 \%$ in the $\chi_{r}$ values is present with the predictions for the $1 \mathrm{~cm}$ and $0.5 \mathrm{~cm}$ grid size case agreeing well with the experimental values. Method 1 performed again poorly in terms of both predicted convective and total heat fluxes, exhibiting a strong grid dependency. It is 


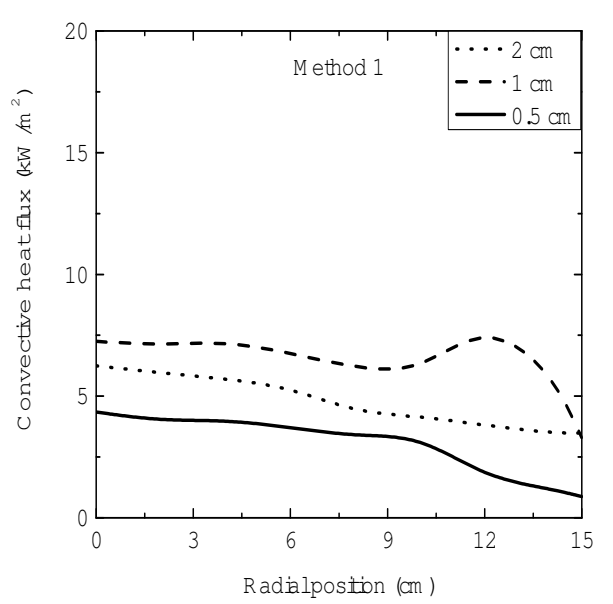

(a)

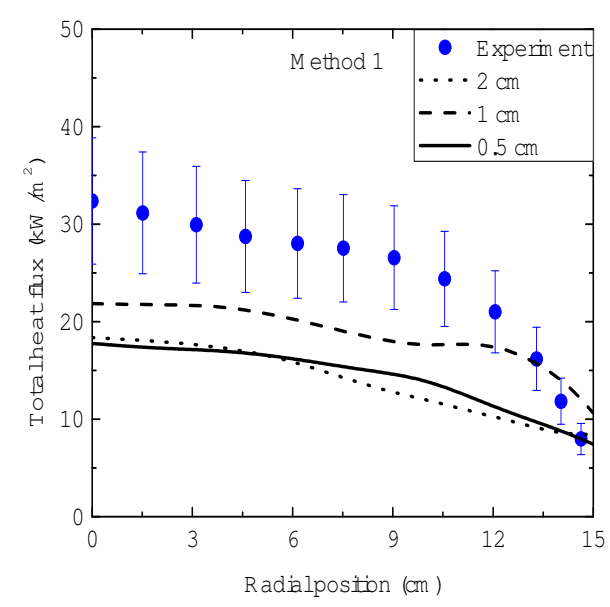

(b)

Fig. 5. Average (a) convective and (b) total heat fluxes at the ethanol $\left(\mathrm{C}_{2} \mathrm{H}_{5} \mathrm{OH}\right)$ pool surface with method 1.

obvious also that any discrepancies in the predicted flow field (i.e., first grid cell values of temperature), due to, e.g., the influence of the pan rim height, result in significant differences in the predicted convective heat fluxes as well (see Figure 5(b)).

\subsection{Acetone}

Finally, the predicted convective and total heat fluxes in the case of the acetone pool fire $\left(\left(\mathrm{CH}_{3}\right)_{2} \mathrm{CO}\right)$ are presented in Figures 6-7 as a function of grid size. Similar observations, like previously made for the other two fuels, can be made here for acetone with method 4 as well regarding the predicted heat fluxes from the simulations. The convective heat fluxes are only slightly grid sensitive. Nevertheless, a grid dependency is present in the total heat fluxes implying that it due to the predictions of the radiative heat fluxes. Typically, the amount of soot emitted from alcohol fires has been reported to vary from little to none for methanol, to some amount for ethanol and somewhat more for acetone [21]. The predicted radiative fraction (calculating the convective heat fluxes with method 4) for the $2 \mathrm{~cm}, 1 \mathrm{~cm}$ and $0.5 \mathrm{~cm}$ test cases were $0.138,0.199$ and 0.208 , respectively, while the experimental was $0.27 \pm 26 \%$ [21]. The discrepancies in the predicted $\chi_{r}$ values are greater in the case of the acetone as opposed to methanol and ethanol reported previously. It is believed that the under-prediction of the radiative fractions in this case, particularly for the $1 \mathrm{~cm}$ and $0.5 \mathrm{~cm}$ grid sizes, are mainly due to the absence of soot modelling in the simulations. Overall, the predictions with method 1 are poor. The fuel region just above the pool surface in the case of the acetone pool fire is much richer, compared to the other two cases, resulting in predicted convective fluxes which are significantly low. 


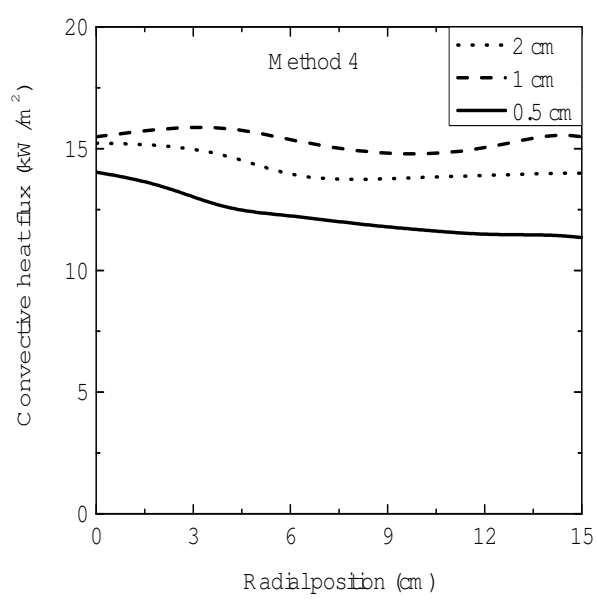

(a)

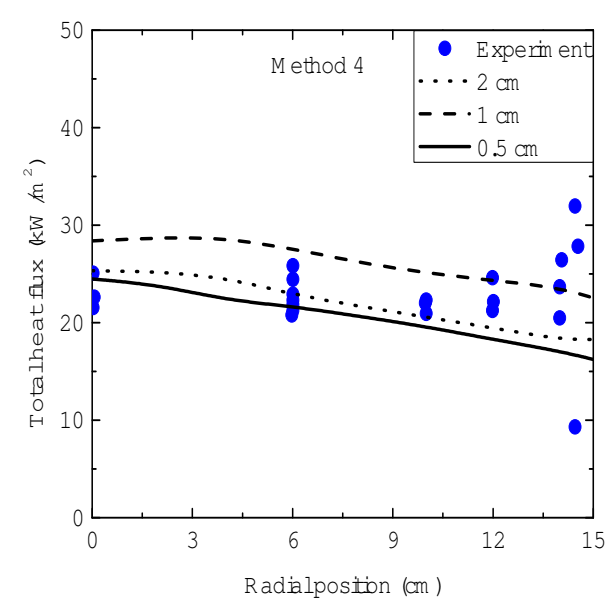

(b)

Fig. 6. Average (a) convective and (b) total heat fluxes at the acetone $\left(\left(\mathrm{CH}_{3}\right)_{2} \mathrm{CO}\right)$ pool surface with method 4.

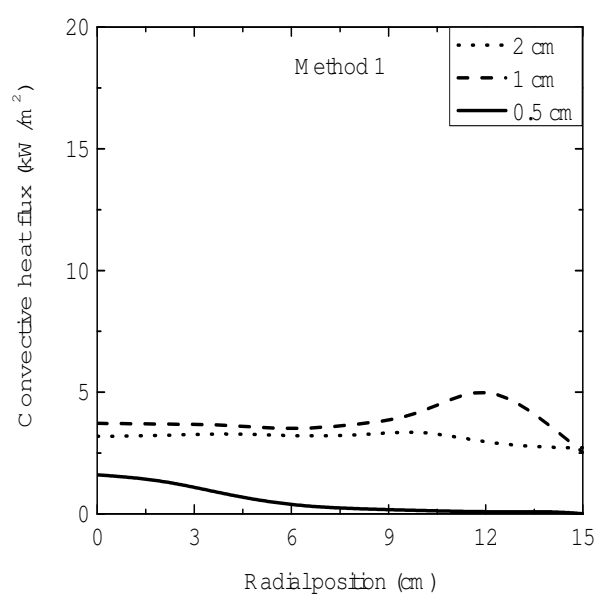

(a)

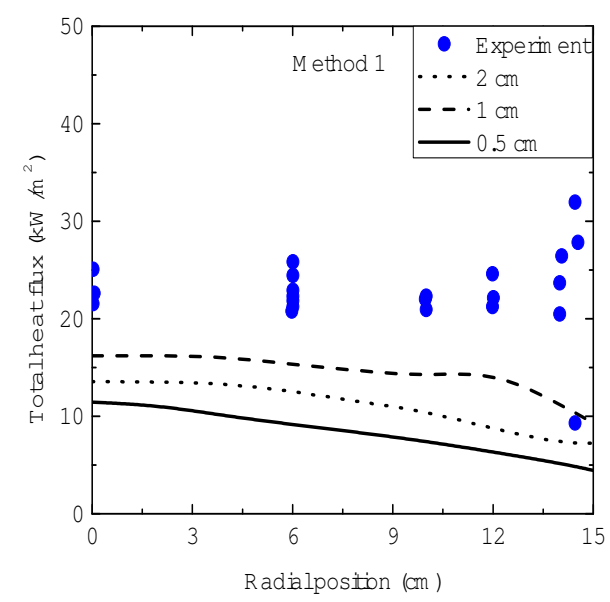

(b)

Fig. 7. Average (a) convective and (b) total heat fluxes at the acetone $\left(\left(\mathrm{CH}_{3}\right)_{2} \mathrm{CO}\right)$ pool surface with method 1.

\section{Conclusions}

Large eddy simulations of medium-scale, $30 \mathrm{~cm}$ in diameter, alcohol pool fires (i.e., methanol, ethanol and acetone) were conducted and the numerical predictions were compared against experimental heat flux measurements [10, 21]. The different methods employed for modelling the convective heat fluxes at the pool surface included an attempt to try to resolve them (method 1), an approach based on a Nusselt correlation for forced convection (method 2), an approach based on a Nusselt correlation for natural convection 
(method 3) and an approach based on the stagnant film theory (method 4).

The numerical results from the present study confirmed that the predictions of the convective heat fluxes at the pool surface can be highly grid dependent when calculated based on methods 1-3, using the first grid cell values, due to a fuel rich region formed just above the pool surface when the grid size is refined. If methods 1-3 are to be employed for modelling convective heat transfer of pool fires, the use of an average flame temperature rather than using the first grid cell temperature would result in more accurate and less grid-sensitive predictions. Overall, the best approach was the one based on the stagnant film theory (method 4) which was only slightly grid sensitive and provided satisfactory predictions for all the different fuels tested. Given its simplicity and robustness, which does not require many a-priori input parameters and can take into account the 'blowing' effect, method 4 is very promising approach to be used in CFD codes for modelling convective heat transfer at the surface of pool fires.

\section{Acknowledgments}

This research has been funded by Ghent University (Belgium) through GOA project BOF16/GOA/004.

\section{References}

[1] S.R. Tieszen, On the fluid mechanics of fires, Annu. Rev. Fluid Mech 33 (2001) 67-92.

[2] P. Joulain, The behavior of pool fires: state of the art and new insights, Proc. Comb. Inst. 27 (1998) 2691-2706.

[3] T. Sikanen, S. Hostikka, Modeling and simulation of liquid pool fires with in-depth radiation absorption and heat transfer, Fire Saf. J. 80 (2016) 95-109.

[4] N. Ren, Y. Wang, S. Vilfayeau, A. Trouvé, Large eddy simulation of turbulent vertical wall fires supplied with gaseous fuel through porous burners, Combust. Flame 169 (2016) 194-208.

[5] K. Li, S. Hostikka, Embedded flame heat flux method for simulation of quasi-steady state vertical flame spread, Fire Saf. J. 104 (2019) 117-129.

[6] K. McGrattan, S. Hostikka, R. McDermott, J. Floyd, M. Vanella, Fire Dynamics Simulator Technical Reference Guide Volume 1: Mathematical Model, NIST Special Publication 1018-1, Sixth Edition, 2019.

[7] ISIS Version 5: Physical Modelling, available through: https://gforge.irsn.fr/gf/project/isis/docman/Physical modelling/

[8] ANSYS FLUENT Theory Guide, Release 15, ANSYS Inc., 2013 
[9] F.P. Incropera, D.P. DeWitt, T.L. Bergman, A.S. Lavine, Fundamentals of Heat and Mass Transfer, 6th Edition, John Wiley \& Sons, New York, 2006.

[10] A. Hamins, S.J. Fischer, T. Kashiwagi, M.E. Klassen, J.P. Gore, Heat Feedback to the Fuel Surface in Pool Fires, Combust. Sci. Technol. 97 (1994) 37-62.

[11] T. Steinhaus, S. Welch, R.O. Carvel, J.L. Torero, Large-scale pool fires, Therm. Sci., 11 (2007) 101-118.

[12] J.G. Quintiere, Fundamentals of fire phenomena, John Wiley \& Sons, New York, 2006.

[13] L. Hu, A review of physics and correlations of pool fire behaviour in wind and future challenges, Fire Saf. J. 91 (2017) 41-55.

[14] https://github.com/fireFoam-dev

[15] G: Maragkos, B. Merci, Large eddy simulations of flame extinction in a turbulent line burner, Fire Saf. J. 105 (2019) 216-226.

[16] J. Welty, G.L. Rorrer, D.G. Foster, Fundamentals of Momentum, Heat and Mass Transfer, John Wiley \& Sons, New York, 2014.

[17] W.H. McAdams, Heat Transmission, Third Edition, McGraw-Hill Book Company, New York, 1957.

[18] D. Drysdale, An Introduction to Fire Dynamics, John Wiley \& Sons, New York, 2011.

[19] L. Orloff, J. De Ris, Froude modeling of pool fires, Proc. Comb. Inst. 19 (1982) 885-895.

[20] A.P. Hamins, J.C. Yang, T. Kashiwagi, Global Model for Predicting the Burning Rates of Liquid Pool Fires, NIST Interagency/Internal Report (NISTIR) - 6381, 1999.

[21] S.C. Kim, K.Y. Lee, A. Hamins, Energy Balance in Medium-Scale Methanol, Ethanol, and Acetone Pool Fires, Fire Saf. J. 107 (2019) 44-53.

[22] A. Hamins, Energetics of Small and Moderate-Scale Gaseous Pool Fires, NIST Technical Note 1926, 2016.

[23] A. Nasr, S. Suard, H. El-Rabii, L. Gay, J.-P. Garo, Fuel Mass-Loss Rate Determination in a Confined and Mechanically Ventilated Compartment Fire Using a Global Approach, Combust Sci. Technol. 183 (2011) 1342-1359.

[24] S. Suard, A. Nasr, S. Melis, J.P. Garo, H. El-Rabii, L. Gay, L. Rigollet, L. Audouin, Analytical Approach for Predicting Effects of Vitiated Air on the Mass Loss Rate of Large Pool Fire in Confined Compartments, Fire Safety Science 10 (2011) 1513-1524.

[25] G. Maragkos, T. Beji, B. Merci, Towards predictive simulations of gaseous pool fires, Proc. Combust. Inst. 37 (2019) 3927-3934. 
[26] E.J. Weckman, A.B. Strong, Experimental Investigation of the Turbulence Structure of Medium-Scale Methanol Pool Fires, Combust. Flame 105 (1996) 245-266.

[27] A.P. Hamins, A. Lock, The Structure of a Moderate-Scale Methanol Pool Fire, Technical Note (NIST TN) - 1928, 2016. 


\section{Figure captions}

Fig. 1. Average radiative heat fluxes at the methanol $\left(\mathrm{CH}_{3} \mathrm{OH}\right)$ pool surface as a function of grid size with (1) method 1, (b) method 2, (c) method 3 and (d) method 4.

Fig. 2. Average convective heat fluxes at the methanol $\left(\mathrm{CH}_{3} \mathrm{OH}\right)$ pool surface as a function of grid size with (1) method 1, (b) method 2, (c) method 3 and (d) method 4.

Fig. 3. Average methanol $\left(\mathrm{CH}_{3} \mathrm{OH}\right)$ mass fraction at the pool surface with grid sizes of (1) $2 \mathrm{~cm}$, (b) $1 \mathrm{~cm}$ and (c) $0.5 \mathrm{~cm}$, calculating the convecting heat fluxes with method 4 .

Fig. 4. Average (a) convective and (b) total heat fluxes at the ethanol $\left(\mathrm{C}_{2} \mathrm{H}_{5} \mathrm{OH}\right)$ pool surface with method 4 .

Fig. 5. Average (a) convective and (b) total heat fluxes at the ethanol $\left(\mathrm{C}_{2} \mathrm{H}_{5} \mathrm{OH}\right)$ pool surface with method 1.

Fig. 6. Average (a) convective and (b) total heat fluxes at the acetone $\left(\left(\mathrm{CH}_{3}\right)_{2} \mathrm{CO}\right)$ pool surface with method 4 .

Fig. 7. Average (a) convective and (b) total heat fluxes at the acetone $\left(\left(\mathrm{CH}_{3}\right)_{2} \mathrm{CO}\right)$ pool surface with method 1. 